\title{
Ajuste y selección de modelos matemáticos para predecir el volumen fustal de Tectona grandis L. f. en Nuevo Urecho, Michoacán, México
}

\section{Selection and adjustment of mathematical models to predict the stem volume of Tectona grandis L. f. in Nuevo Urecho, Michoacan, Mexico}

\author{
Ricardo Telles Antoniol*, Martín Gómez Cárdenas², Eduardo Alanís Rodríguezl, \\ Oscar Alberto Aguirre Calderón' y Javier Jiménez Pérez'
}

Universidad Autónoma de Nuevo León. Facultad de Ciencias Forestales. Linares, Nuevo León, México. 2 Instituto Nacional de Investigaciones Forestales
Agrícolas y Pecuarias. Campo Experimental
Uruapan, CIR-Pacífico Centro. Uruapan, Michoacán,
México.
* Autor de correspondencia. telles.ricardo2015@ gmail.com

\section{RESUMEN}

El objetivo fue ajustar y seleccionar un modelo matemático para predecir el volumen fustal de Tectona grandis L. f. en una plantación de 11 años de edad en Nuevo Urecho, Michoacán. La muestra analizada fue de 128 árboles, a los que se les midió el diámetro de tocón, diámetro normal y el diámetro a distintas secciones, desde la altura de tocón hasta altura total. Se ajustaron seis modelos de volumen fustal mediante el procedimiento Model del paquete estadístico SAS 9.2®. La selección del modelo se hizo a partir de la suma de

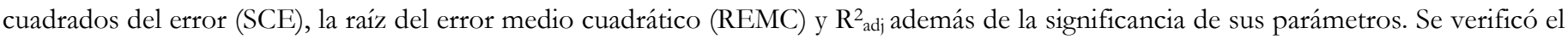
cumplimiento de los supuestos de normalidad, homogeneidad de varianzas e independencia de la frecuencia de residuos. Se generó un criterio de calificación que jerarquizó cada estadístico en función al orden de importancia, siendo mejores aquellos con el valor más bajo en la calificación total. El modelo de Meyer fue el de mejor ajuste con SCE=0.0399, REMC $=0.0179$ y R ${ }^{2}$ adj $=0.8246$. La prueba de Shapiro-Wilk (0.97), White's Test (13.00) y la de Durbin-Watson (2.0112) no violan los supuestos de la regresión y garantizan la eficacia del modelo para la estimación de la variable dependiente. Este modelo se considera apropiado para predecir con mayor precisión el volumen fustal en función del diámetro normal (d) y la altura total (AT) de T. grandis en plantaciones con condiciones físicas y biológicas similares a las del sitio de este estudio.

PALABRAS CLAVE: biometría, ecuaciones, madera, plantaciones comerciales, silvicultura.

\section{ABSTRACT}

The objective was to adjust and select a mathematical model to predict the stem volume of Tectona grandis L. f. in an 11-year-old plantation in Nuevo Urecho, Michoacan. The sample analyzed was 128 trees, in which it was measured the stump diameter, normal diameter and diameters to different sections, from stump height to total height. Six stem volume models were adjusted using the SAS 9.2® statistical package Model procedure. The selection of the model was made from the total square error (SCE), the root mean square error (REMC) and $\mathrm{R}^{2}$ adj as well as the significance of their parameters. Compliance with the assumptions of normality, homogeneity of variances and independence of waste frequency was verified. A rating criterion was generated that ranked each statistics according to the order of importance, with the best being those with the lowest value in the overall rating. Meyer's model was the best fit with SCE $=0.0399$, $\mathrm{REMC}=0.0179$ and $\mathrm{R}^{2}$ adj $=0.8246$. The Shapiro-Wilk test (0.97), White's Test (13.00), Durbin-Watson (2.0112) do not violate the regression assumptions and guarantee the effectiveness of the model for the estimation of the dependent variable. This model is considered appropriate to predict more accurately the stem volume as a function of the normal diameter (d) and total height (AT) of T. grandis in plantations with physical and biological conditions similar to those of the site of this study.

KEYWORDS: biometrics, equations, wood, commercial plantations, forestry. 


\section{INTRODUCCIÓN}

Una de las principales características de las plantaciones forestales comerciales (PFC) que inciden en la toma de decisiones en el manejo forestal es el volumen maderable. Con la finalidad de evaluar el valor de las cosechas se necesita conocer a detalle los volúmenes de los fustes (Hynynen, 2011). Una especie forestal exótica importante en las PFC en México es la teca (Tectona grandis L. f.), la cual se ha adaptado bien en regiones del centro y sur, específicamente en los estados de Campeche, Chiapas, Tabasco, Veracruz, Michoacán y Nayarit. Se calcula que la superficie establecida de PFC de teca en México es de 25,324 hectáreas (Comisión Nacional Forestal [Conafor], 2014).

Los modelos matemáticos construidos con relaciones alométricas permiten estimar una determinada variable como el diámetro normal, el diámetro de copa, la altura total, el volumen, la biomasa o el carbono que serían de difícil y costosa medición en campo; estos modelos reducen la cantidad de trabajo y esfuerzo requeridos para cuantificarlas, en función de variables de fácil medición como el diámetro de tocón, diámetro normal o la altura total. Cuando se hace referencia al crecimiento en volumen en plantaciones forestales, normalmente la expresión se refiere al volumen fustal (y no al volumen total del árbol, incluyendo raíces, ramas y follaje); por lo tanto, este crecimiento en volumen está en función directa del crecimiento directa del crecimiento en altura y en diámetro (Vallejo y Avendaño, 2013).

Los modelos de volumen para teca desarrollados por Pérez y Kanninen (2007) sirven como ejemplo de este tipo de enfoque de modelación; estos autores construyeron un modelo para el volumen fustal a partir del diámetro normal y la altura de los árboles como variables independientes. Con este sistema es posible predecir el volumen de madera en árboles de una manera flexible (Hynynen, 2011). En Latinoamérica se han desarrollado modelos alométricos aplicables a la especie y son utilizados con frecuencia en los inventarios forestales, junto con los modelos de crecimiento, como base para la estimación de las existencias en pie y para el manejo de plantaciones. Camacho-Linton, Ramírez-Maldonado, de los Santos-Posadas y Zamudio-
Sánchez (2013) calcularon el volumen fustal de árbol individual por medio de la ecuación de Pérez y Kanninen (2007) en plantaciones de teca en el estado de Campeche. En Costa Rica, Camacho y Madrigal (1997), ajustaron el modelo de la variable combinada para predecir el volumen de fuste en plantaciones con diámetros de $5 \mathrm{~cm}$ a $30 \mathrm{~cm}$; en Colombia, Torres (2004), ajustó y seleccionó modelos para plantaciones de 12 años de edad; y Corona, Jacas, Tamayo, y Pereira (1999) en Cuba, seleccionaron modelos para estimar el volumen de fuste de árboles de 25 años de edad. En México Tamarit et al. (2014) elaboraron un sistema de cubicación de árbol individual para plantaciones de teca en el sureste mexicano, integrado por modelos de volumen total y comercial variable generados a partir de funciones segmentadas de ahusamiento.

\section{OBJETIVOS}

Ajustar y seleccionar un modelo matemático para predecir volumen fustal de teca (T. grandis L. f.) en una plantación a los 11 años de establecida en Nuevo Urecho, Michoacán.

\section{MATERIALES Y MÉTODOS}

\section{Área de estudio}

El estudio se realizó en una PFC de Tectona grandis localizada en el paraje "El Mirador", municipio de Nuevo Urecho, Michoacán. Este sitio se encuentra en el Eje Neovolcánico Transversal que comprende los cerros El Tipítaro, de las Gallinas, de Agua Fría y de las Cuevas (Instituto Nacional de Estadística y Geografía [Inegi], 2009). Está delimitado por las coordenadas geográficas de latitud N 19¹1'39.6" y longitud W 101 $51^{\prime} 53.3^{\prime \prime}$, a una altitud promedio de $617 \mathrm{~m}$ y presenta una pendiente de $1 \%$ a $2 \%$. Los árboles están plantados con un espaciamiento de $2.0 \mathrm{~m} \times 3.0 \mathrm{~m}$, que corresponde a una densidad de 1666 árboles/ha. Los suelos predominantes son Vertisol (38.32\%), Luvisol (32.24\%), Leptosol (26.08\%), Phaeosem (2.84\%), Fluvisol (0.28\%) y Regosl (0.03\%) (Inegi, 2009). El clima es Awo(w), cálido subhúmedo con lluvias en verano, de humedad media (91.16\%); temperatura media anual de $20{ }^{\circ} \mathrm{C}$ a $28{ }^{\circ} \mathrm{C}$ y de precipitación anual de $700 \mathrm{~mm}$ a $1100 \mathrm{~mm}$ (Inegi, 2009). 
Dentro del área de estudio, se seleccionó el arbolado con base en la metodología propuesta por Segura y Andrade (2008), quienes indican que los individuos seleccionados para medir deben ser "típicos" de la especie y del sitio, describen que el término "típico" se refiere a que los individuos deben tener la forma y sanidad del fuste de la población muestreada. Dentro de los seis círculos se seleccionaron algunos árboles sin daños ni defectos, cubriendo la mayor cantidad de clases diamétricas y de altura posible, como lo indican Barrios, López y Nieto (2014). Con respecto al número de árboles, se siguió lo recomendado por Louppe y Mille (2015), quienes mencionan que se midan unos 100 árboles en caso de uno o varios rodales de plantación reciente en una superficie restringida. (Fig. 1).

\section{Datos dasométricos}

La toma de datos de campo se realizó en el año 2014, a los 11 años de edad de la plantación. Se utilizó un muestreo selectivo de árboles con las condiciones y variaciones en sus medidas para un ajuste adecuado. En total se midieron 128 árboles, a los cuales se colectaron indirectamente datos de diámetros a $0.3 \mathrm{~m}$ sobre el nivel del suelo $\left(\mathrm{d}_{0.3}\right)$, diámetro a la altura de $1.3 \mathrm{~m}\left(\mathrm{~d}_{1.3}\right)$ y los demás diámetros a cada dos metros $\left(\mathrm{d}_{2}, \mathrm{~d}_{4}, \mathrm{~d}_{6}, \mathrm{~d}_{8}, \mathrm{~d}_{10}, \mathrm{~d}_{12}, \mathrm{~d}_{14}\right)$ hasta la altura total (AT) con el Dendrómetro Criterion RD $1000 \AA$. Para la cubicación de trozas se formaron secciones de dos metros de largo, con diámetro menor y mayor conocidos, con lo cual fue calculado el volumen fustal por el método de Smalian (Ec.

1) (De Cesaro, Engel, Finger y Schneider, 1994).

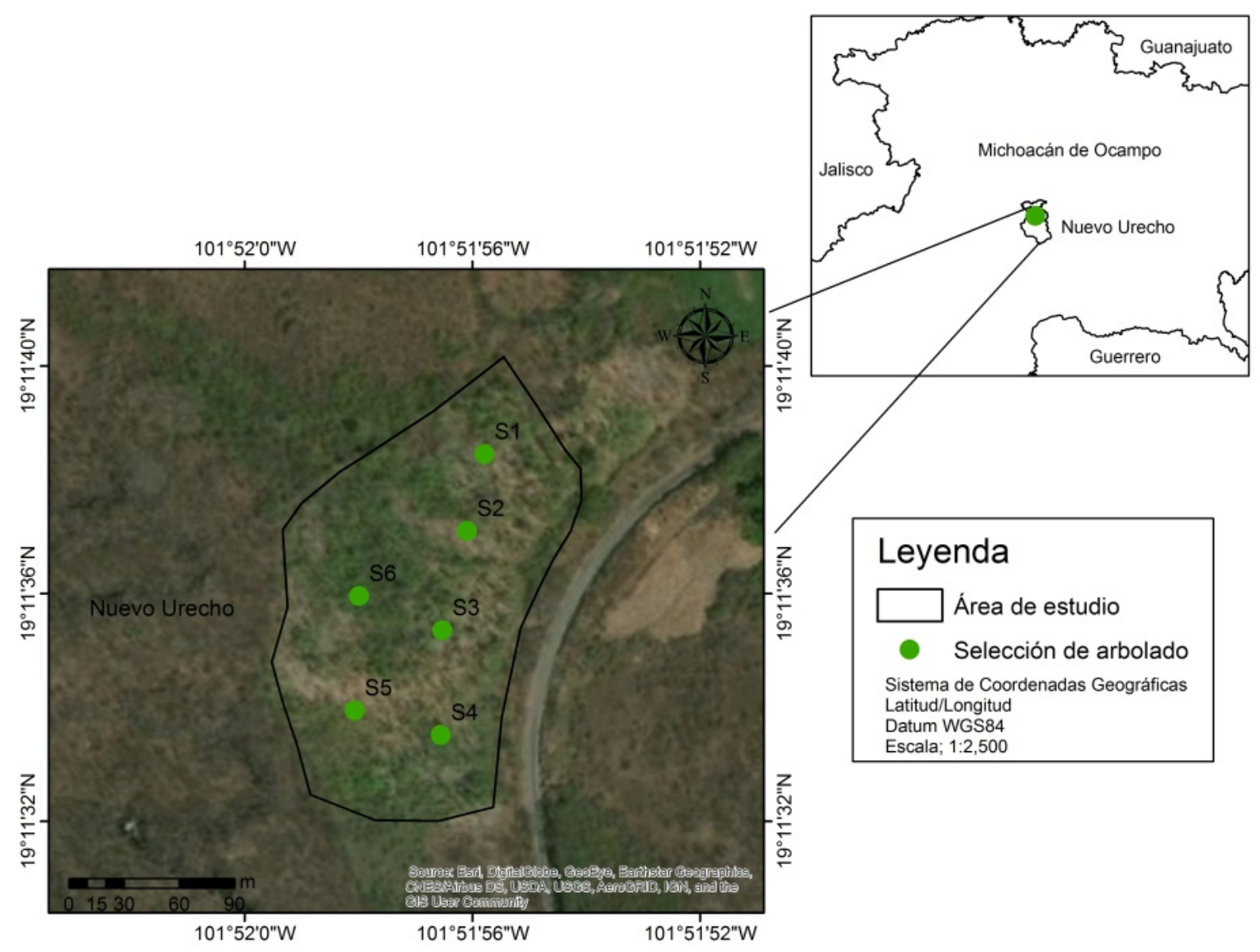

FIGURA 1. Localización del área de estudio y selección de arbolado en la plantación de Tectona grandis en Nuevo Urecho, Michoacán. 


$$
\mathrm{V}_{\mathrm{i}}=\sum_{\mathrm{i}=1}^{\mathrm{n}} \frac{\mathrm{gi+gi}}{2} \cdot \mathrm{L}_{\mathrm{i}}
$$

donde:

$\mathrm{V}_{\mathrm{i}}=$ Volumen de las secciones intermediarias $\left(\mathrm{m}^{3}\right)$

gi $=$ Sección transversal en la i-ésima posición $\left(\mathrm{m}^{2}\right)$

$\mathrm{L}_{\mathrm{i}}=$ Largo de la sección en la i-ésima posición $(2 \mathrm{~m})$

Para la obtención del volumen fustal con corteza individual fue sumado el volumen de cada sección (Ec. 2), más el volumen de cono (Ec. 3) formado por la última sección.

$$
\mathrm{V}=\mathrm{V}_{0}+\sum_{\mathrm{i}=1}^{\mathrm{n}} \mathrm{V}_{\mathrm{i}}+\mathrm{V}_{\mathrm{c}}
$$

donde:

$\mathrm{V}=$ Volumen fustal con corteza $\left(\mathrm{m}^{3}\right)$.

$\mathrm{V}_{\mathrm{o}}=$ Volumen del tocón $\left(\mathrm{m}^{3}\right)$ hasta 0.3 metros desde el nivel del suelo.

$\mathrm{V}_{\mathrm{c}}=$ Volumen de la punta superior del árbol $\left(\mathrm{m}^{3}\right)$ calculado por la ecuación del cono, de largo igual a la sección considerada, donde:

$$
\mathrm{V}_{\mathrm{c}}=\mathrm{g}_{\mathrm{n}} \cdot \mathrm{L}_{\mathrm{n}} \cdot \frac{1}{3}
$$

donde:

$\mathrm{g}_{\mathrm{n}}=$ Área de la sección transversal mayor del cono $\left(\mathrm{m}^{2}\right)$

$\mathrm{L}_{\mathrm{n}}=$ Altura del cono $(\mathrm{m})$

\section{Modelos de volumen fustal}

Para elegir el modelo que represente la verdadera relación entre las variables, Husch, Beers y Kershaw Jr. (2002) recomiendan verificar la forma de la línea o curva del conjunto de datos, mediante una gráfica de puntos (criterio sólido). Como el trazado de puntos es considerado un criterio que incide en la elección de modelos cuando se conoce poco del tema, se optó por verificar la forma de la línea que resulta de las variables de Tectona grandis, generando una gráfica de dispersión, que permitió ver la tendencia de datos y el comportamiento de las variables independientes respecto a la variable dependiente; de tal forma que el modelo tendrá que predecir tal tendencia para facilitar su ajuste (Reyes, 2006).

De la elección del tipo de modelo dependerá la precisión de la estimación mediante la ecuación, ya que el método de mínimos cuadrados garantiza que la curva sea la mejor ajustada, pero esto no quiere decir que el modelo sea el apropiado (Reyes, 2006).

En el estudio se seleccionaron para su evaluación seis modelos de volumen fustal (M1 a M6) (Tabla 1), la estimación de los parámetros en el ajuste de los modelos se llevó a cabo con métodos iterativos (Draper y Smith, 2014), con el procedimiento Model y el algoritmo Gauss-Newton del paquete estadístico SAS 9.2® (Allison, 2010), mediante el método de ajuste de mínimos cuadrados ordinarios (MCO).

TABLA 1. Modelos matemáticos evaluados para la estimación del volumen fustal para Tectona grandis en Nuevo Urecho, Michoacán.

\begin{tabular}{cccc}
\hline Variable Independiente & No. & Modelo & Expresión matemática \\
\hline & M1 & Burkhart (1977) & $V=\beta_{0}+\beta_{1} \cdot d^{\beta 2} \cdot H^{\beta 3}+\varepsilon$ \\
& M2 & Australiana & $V=\beta_{0}+\beta_{1} \cdot d^{2}+\beta_{2} \cdot H+\beta_{3} \cdot d^{2} \cdot H$ \\
M3 & Stoate (1945) & $V=\beta_{0}+\beta_{1} \cdot d^{2}+\beta_{2} \cdot d^{3}+\beta_{3} \cdot H+\beta_{4} / H+\varepsilon$ \\
M4 & Meyer (1941) & $V=\beta_{0}+\beta_{1} \cdot d+\beta_{2} \cdot d^{2}+\beta_{3} \cdot d \cdot H+\varepsilon$ \\
& M5 & Prodan, Peters, Cox y Real (1997) & $V=\beta_{0}+\beta_{1} \cdot d^{2} \cdot H+\beta_{2} \cdot d^{2}+\varepsilon$ \\
& M6 & Naslud (1936) & $V=\beta_{1}+\beta_{2} \cdot d^{2}+\beta_{3} \cdot d^{2} \cdot H+\beta_{4} \cdot H^{2}+\beta_{5} \cdot D \cdot H^{2}$ \\
\hline
\end{tabular}

d: diámetro a la altura de $1.3(\mathrm{~m}), \mathrm{H}$ : altura total $(\mathrm{m}), \mathrm{V}$ : volumen fustal $\left(\mathrm{m}^{3}\right)$, $\beta$ i: parámetros a ser estimados y $\varepsilon$ : error aleatorio del modelo. 


\section{Criterios de evaluación}

La selección del mejor modelo se hizo a partir de la bondad de ajuste, medida con la suma de cuadrados del error (SCE), la raíz del error medio cuadrático (REMC) (Ec. 4), el coeficiente de determinación ajustado por el número de parámetros del modelo ( $\mathrm{R}^{2}$ adj), y la significancia de los parámetros ( Schlaegel, 1982; Parresol, 1999; Tedeschi, 2006).

$$
\mathrm{REMC}=\sqrt{\frac{\sum_{\mathrm{i}=1}^{\mathrm{n}}\left(\mathrm{Y}_{\mathrm{i}} \hat{\mathrm{Y}}_{\mathrm{i}}\right)^{2}}{\mathrm{n}-\mathrm{p}}}
$$

\section{Validación del modelo seleccionado}

Cuando las varianzas de la frecuencia de los residuos son heterogéneas (heterocedasticidad) o cuando los residuos están autocorrelacionados, las estimaciones de los coeficientes de regresión por el método de los mínimos cuadrados ordinarios son adversamente afectadas y la estimación del error estándar es tendenciosa. Esto justifica la validación de la ecuación seleccionada para la verificación de dichos supuestos, el cual indica la calidad de la predicción.

Para verificar el cumplimiento de los supuestos de la regresión se determinaron la normalidad de los residuos, la homogeneidad de varianzas de los residuos y la independencia de los residuos:

a) Normalidad de los residuos. Se calculó la estadística de Shapiro-Wilk (Ec. 5) (Da Cunha, Vargas y Escalier, 2009).

$$
\mathrm{W}_{\mathrm{c}}=\frac{\mathrm{b}^{2}}{\sum_{\mathrm{i}=1}^{\mathrm{n}}\left(\mathrm{X}_{\mathrm{i}}-\hat{\mathrm{y}}\right)^{2}}
$$

b) La homogeneidad de varianzas de los residuos; una de las principales presuposiciones para los mínimos cuadrados de la regresión usual es la homogeneidad de varianza (homocedasticidad) (Emanuelli y Milla, 2014). El método matemático para determinar si hay homogeneidad de varianza de los residuos fue mediante la prueba de White.

c) Independencia de los residuos; es esperado que la estadística “ " $d$ ” (Ec. 6), sea aproximadamente igual a 2, si los residuos son independientes. Caso contrario, si los residuos son correlacionados positivamente, tenderán a ser próximos de 0 (cero), o próximos a 4, si los residuos son correlacionados negativamente (Emanuelli y Milla, 2014). El valor de “ $d$ ” será dado por:

$$
\mathrm{d}=\frac{\sum_{\mathrm{i}=2}^{\mathrm{n}}\left(\mathrm{E}_{\mathrm{i}}-\mathrm{E}_{\mathrm{i}-1}\right)^{2}}{\sum_{\mathrm{i}=1}^{\mathrm{n}} \mathrm{E}_{\mathrm{i}}^{2}}
$$

donde:

$d=$ estadística " $d$ " de Durbin-Watson;

$n=$ número de observaciones;

$E_{i}=$ error estocástico

Se generó un criterio de calificación que de acuerdo con Sakici, Misir, Yavuz y Misir (2008), que consistió en jerarquizar cada estadístico de cada modelo asignando valores consecutivos del 1 al 6 en función del orden de importancia (1 correspondió al mejor valor del estadístico y 6 al valor más pobre). Posteriormente, la sumatoria de los valores conformó la calificación total de cada modelo. Por comparación se identificaron los mejores modelos, siendo mejores aquellos con el valor más bajo en la calificación total.

Se analizó la distribución de los residuales mediante método gráfico de las ecuaciones de regresión de mejor ajuste para Tectona grandis, para observar la distribución de los residuos (diferencias entre valor observado y valor predicho), tal como lo recomiendan Álvarez, Barrio, Diéguez y Rojo (2003), ya que mencionan que de esta forma se evidencia alguna deficiencia de los modelos ajustados.

\section{Resultados}

Con base en el conjunto de datos de los 128 árboles de $T$. grandis, en la tabla 2 se presentan las estadísticas básicas de las variables analizadas; los valores de los parámetros de cada uno de los modelos ajustados, así como sus indicadores de bondad de ajuste, nivel de confiabilidad (Tabla 3) y la calificación resultante (Tabla 4).

Se calculó el volumen de cada árbol muestreado, previo al análisis de los datos, el comportamiento y tendencias de las variables (diámetro normal, altura total y volumen acumulado) fueron inspeccionados de forma gráfica, lo que permitió corregir y depurar la base de datos (Fig. 2). 
TABLA 2. Estadísticos de las variables medidas en árboles de Tectona grandis.

\begin{tabular}{lcccc}
\hline Variable & Máximo & Medio & Mínimo & Desviación Estándar \\
\hline Diámetro tocón $(\mathrm{cm})$ & 35.90 & 22.95 & 14.80 & 3.67 \\
Diámetro normal $(\mathrm{cm})$ & 24.20 & 16.55 & 11.60 & 2.41 \\
Altura total $(\mathrm{m})$ & 17.60 & 13.01 & 8.50 & 1.69 \\
\hline
\end{tabular}

TABLA 3. Valores de los estadísticos de bondad de ajuste de los seis modelos evaluados en la estimación del volumen fustal de Tectona grandis.

\begin{tabular}{|c|c|c|c|c|c|c|c|c|}
\hline No & Modelo & $R_{a d j}^{2}$ & SCE & REMC & $\overline{\beta_{i}}$ & Valor Estimado & Error Estándar & Prob $>T$ \\
\hline \multirow[t]{4}{*}{1} & Burkhart & 0.8248 & 0.0398 & 0.0179 & $\beta_{0}$ & 0.038774 & 0.0131 & 0.0038 \\
\hline & & & & & $\beta_{l}$ & 0.000008 & 0.000012 & 0.4807 \\
\hline & & & & & $\beta_{2}$ & 2.472970 & 0.3338 & $<0.0001$ \\
\hline & & & & & $\beta_{3}$ & 0.903432 & 0.2037 & $<0.0001$ \\
\hline \multirow[t]{4}{*}{2} & Australiana & 0.8231 & 0.0402 & 0.0180 & $\beta_{0}$ & 0.048087 & 0.0401 & 0.2330 \\
\hline & & & & & $\beta_{l}$ & -0.000007 & 0.000157 & 0.9641 \\
\hline & & & & & $\beta_{2}$ & 0.000032 & 0.000012 & 0.0072 \\
\hline & & & & & $\beta_{3}$ & -0.002380 & 0.00308 & 0.4415 \\
\hline \multirow[t]{5}{*}{3} & Stoat & 0.8227 & 0.0400 & 0.0180 & $\beta_{0}$ & -0.168480 & 0.1424 & 0.2391 \\
\hline & & & & & $\beta_{1}$ & -0.000020 & 0.000196 & 0.9282 \\
\hline & & & & & $\beta_{2}$ & 0.000017 & 7.49E-06 & 0.0245 \\
\hline & & & & & $\beta_{3}$ & 0.011507 & 0.0053 & 0.0319 \\
\hline & & & & & $\beta_{4}$ & 0.942800 & 0.8627 & 0.2766 \\
\hline \multirow[t]{4}{*}{4} & Meyer & 0.8246 & 0.0399 & 0.0179 & $\beta_{0}$ & 0.127677 & 0.0535 & 0.0184 \\
\hline & & & & & $\beta_{1}$ & -0.018420 & 0.00654 & 0.0056 \\
\hline & & & & & $\beta_{2}$ & 0.000815 & 0.000195 & $<0.0001$ \\
\hline & & & & & $\beta_{3}$ & 0.000374 & 0.000067 & $<0.0001$ \\
\hline \multirow[t]{3}{*}{5} & Prodan & 0.8237 & 0.0404 & 0.0180 & $\beta_{0}$ & 0.017514 & 0.00646 & 0.0077 \\
\hline & & & & & $\beta_{1}$ & 0.000023 & 4.00E-06 & $<0.0001$ \\
\hline & & & & & $\beta_{2}$ & 0.000103 & 0.000066 & 0.1247 \\
\hline \multirow[t]{5}{*}{6} & Naslud & 0.8224 & 0.0401 & 0.0181 & $\beta_{0}$ & 0.026535 & 0.0253 & 0.2965 \\
\hline & & & & & $\beta_{1}$ & -0.000110 & 0.000218 & 0.6128 \\
\hline & & & & & $\beta_{2}$ & 0.000050 & 0.000027 & 0.0637 \\
\hline & & & & & $\beta_{3}$ & -0.000030 & 0.000037 & 0.4325 \\
\hline & & & & & $\beta_{4}$ & 0.000194 & 0.000386 & 0.6159 \\
\hline
\end{tabular}

$R^{2}$ adj: Coeficiente de determinación ajustado. SCE: Suma de cuadrado del error. ßi: Parámetros estimados. REMC: Raíz del error medio cuadrático. 
TABLA 4. Sistema de calificación para los modelos ajustados para predecir el volumen fustal de Tectona grandis.

\begin{tabular}{ccccccc}
\hline Modelo & $R^{2}$ adj & SCE & CME & REMC & Sesgo & Calificación total \\
\hline 1 & 1 & 1 & 1 & 1 & 3 & 7 \\
2 & 4 & 5 & 4 & 4 & 1 & 18 \\
3 & 5 & 3 & 5 & 3 & 2 & 18 \\
4 & 2 & 2 & 2 & 2 & 4 & 12 \\
5 & 3 & 6 & 3 & 5 & 5 & 22 \\
6 & 6 & 4 & 6 & 6 & 6 & 28 \\
\hline
\end{tabular}
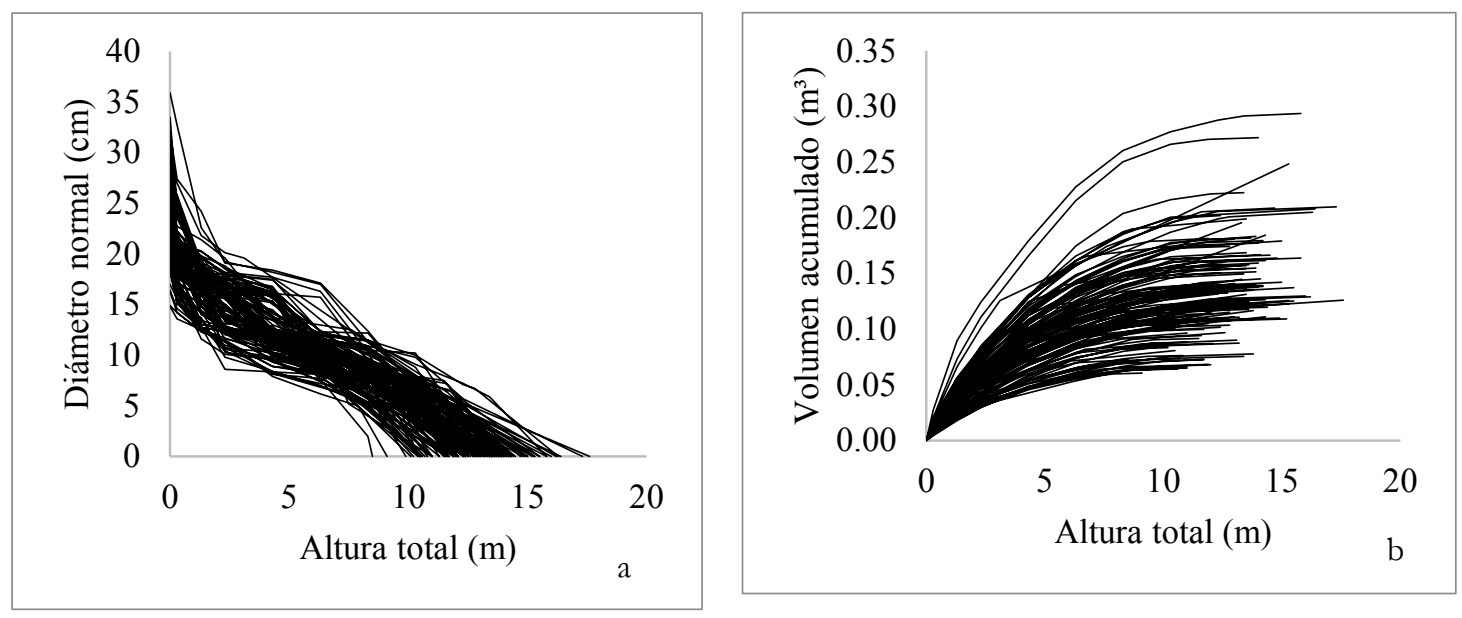

FIGURA 2. Comportamiento del diámetro normal (a) y del volumen fustal acumulado (b) con respecto a la altura total de Tectona grandis.

La distribución de la relación de la variable combinada (diámetro normal y volumen fustal) de Tectona grandis presentada en la figura 3, sustenta el uso de modelos lineales y no lineales, en la estimación del volumen fustal.

Los modelos 1 y 4 (M1, SCE $=0.0398$ y M4, SCE $=0.0399)$ obtuvieron los valores más pequeños con respecto a los otros modelos, los cuáles dan una expresión explícita de los coeficientes estimados (Tabla 3).

Se observa que el M1, presenta los criterios de bondad de ajuste mejor y la calificación total más baja (Tabla 4), sin embargo, el parámetro $\beta_{1}(0.4807)$ no fue significativo por lo que se descarta el ajuste del modelo. Por lo anterior y de acuerdo con los criterios de selección, el M4, que en orden de calificación presenta la más baja, se puede elegir como el mejor.
El M4 explica arriba de $82 \%$ la variabilidad total presente en la variable dependiente, con un valor de REMC $=0.0179$, que es un valor menor con respecto a los demás modelos, menor valor de SCE $=0.0399$ y una alta significancia en cada uno de sus parámetros (Tabla 3); además presenta un sesgo absoluto de $0.0083 \mathrm{~m}^{3}$, lo cual indica que se tiene la mínima desviación del modelo con respecto a los valores observados, por lo que se considera apropiado para predecir con mayor precisión el volumen fustal en función del diámetro normal (d) y la altura total (AT).

Una vez examinado el cumplimiento de los supuestos de la regresión en el M4, la prueba de la normalidad de Shapiro-Wilk, mostró un valor de 0.97 (Tabla 5); los porcentajes de frecuencias relativas acumuladas de los 
residuales muestran una relación cercana a una línea recta, respecto de la probabilidad de la distribución normal, sus porcentajes tienden a formar una campana de Gauss.

La dispersión de los residuos, respecto a los valores estimados de volumen M4 (Fig. 4) no presenta evidencia de alguna deficiencia. La prueba de White obtuvo un valor de 13.00 (Tabla 5), el cual no es significativo en los residuales $(\operatorname{Pr}>C h i-S q=0.119)$, por lo que no existe evidencia estadística significativa de heterocedasticidad.

El resultado del estadístico Durbin-Watson, de independencia de la frecuencia de los residuos demuestra que no hay colinealidad entre variables (Tabla 5). Por lo tanto, la ecuación generada de volumen fustal a partir de los valores de los parámetros de la tabla 3, para el M4, queda definida como:

$V=0.127677+-0.018420 * \mathrm{~d}+0.000815 * \mathrm{~d}^{2}+0.000374 * \mathrm{~d} * \mathrm{H}+\varepsilon$

donde:

$V=\operatorname{Volumen}$ fustal $\left(\mathrm{m}^{3}\right)$

$d=$ Diámetro normal $(\mathrm{cm})$

$H=$ Altura total $(\mathrm{m})$

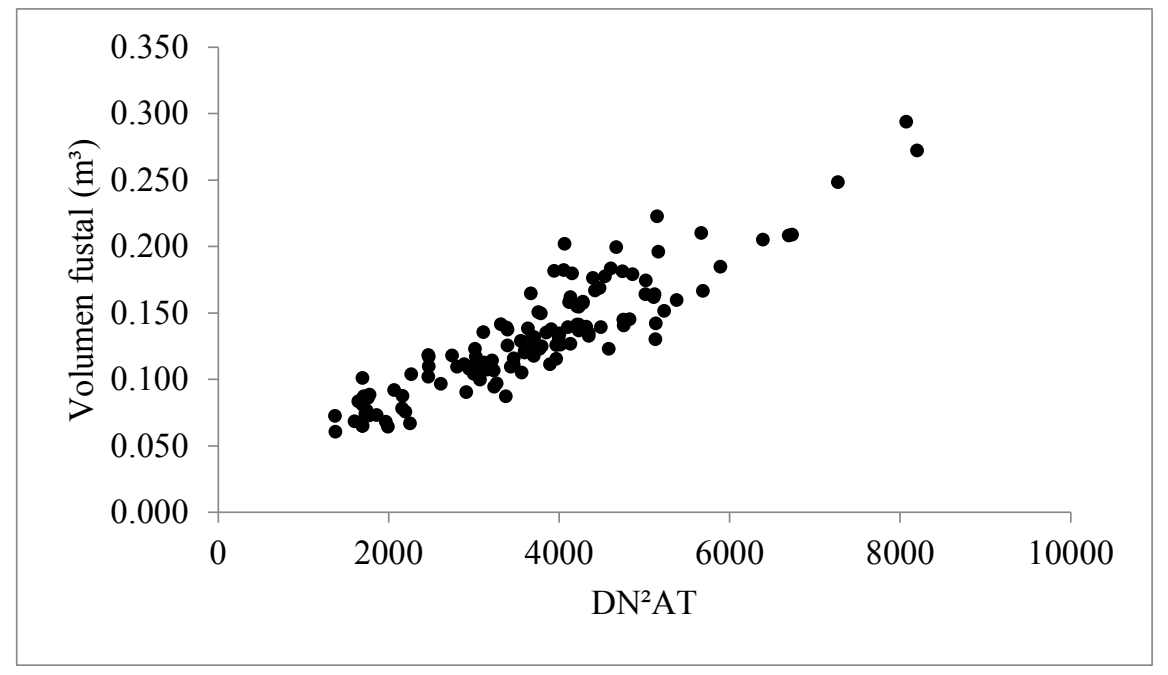

FIGURA 3. Dispersión de datos de la variable combinada (diámetro normal y altura total) y volumen fustal de Tectona grandis.

TABLA 5. Resultados de las pruebas de Shapiro-Wilk, White y Durbin-Watson para el modelo de mejor ajuste para predecir el volumen fustal de Tectona grandis.

\begin{tabular}{cccccc}
\hline Modelo & Shapiro-Wilk & Pr<W & Prueba de White & Pr>ChiSq & $\begin{array}{c}\text { Durbin-Watson } \\
\text { (DW) }\end{array}$ \\
\hline Meyer (1941) & 0.97 & 0.0683 & 13.00 & 0.1119 & 2.0112 \\
\hline
\end{tabular}




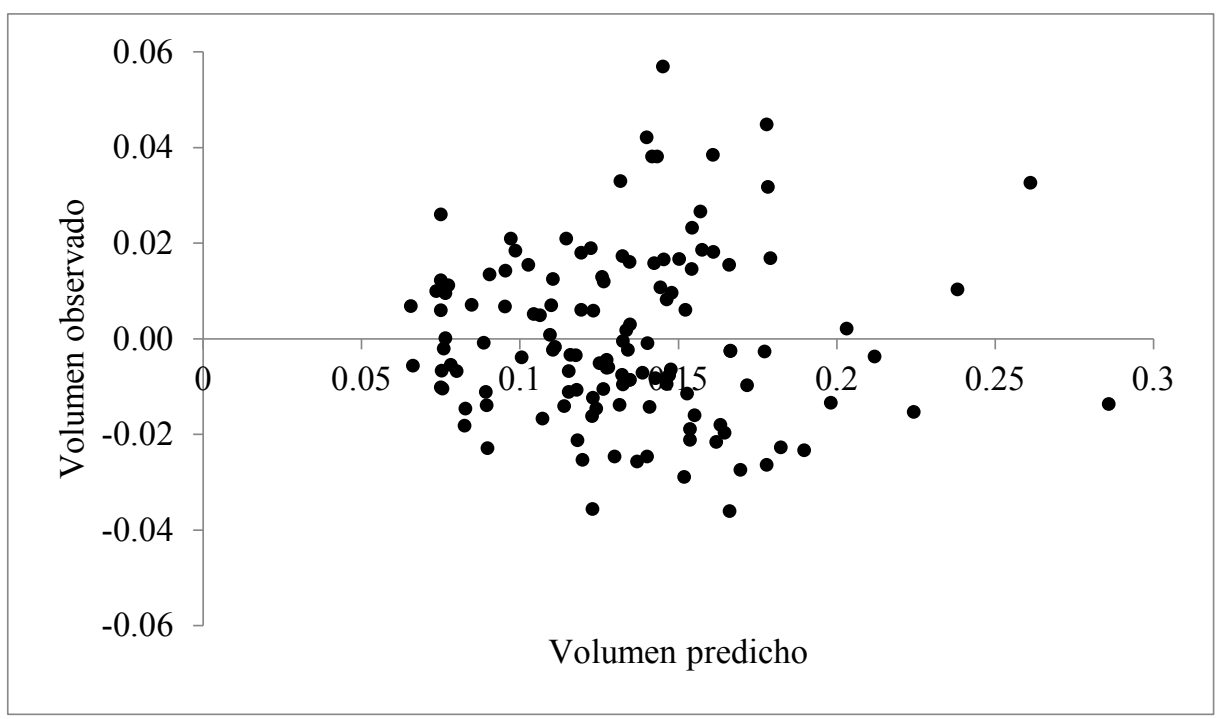

Figura 4. Distribución de residuos del Modelo de Meyer de volumen fustal para Tectona grandis.

\section{DISCUSIÓN}

Con base en los criterios de bondad de ajuste, el $\mathrm{R}^{2}$ adj de los modelos no varió. Se propone el uso del M4, seleccionado con base en el sistema de calificación empleado, el cual presenta la más alta precisión en las estimaciones dado su valor menor de REMC y corrobora que el modelo predice satisfactoriamente el volumen fustal. El sesgo como indicador de la capacidad de estimación del modelo subestima el volumen fustal, su evolución se mantiene más cercana a cero en todas las categorías diamétricas. El resultado de la prueba de independencia de los residuos coincide con Da Cunha et al. (2009), quienes concluyen que un valor más cercano a 2 en dicha prueba, no viola los supuestos de la regresión y es más preciso para la estimación de la variable dependiente.

El M4 con base en el valor de ajuste de $\mathrm{R}^{2}$ adj concuerda con lo hallado por Corona et al. (1999), quienes ajustaron un modelo de volumen donde emplearon como variables predictoras el diámetro y la altura y, obtuvieron un valor de $\mathrm{R}^{2}$ adj de 0.80 para plantaciones de teca a la edad de 25 años establecidas en la Unidad Silvícola Guisa, Cuba.

El modelo seleccionado en este estudio, para la plantación de teca en Michoacán, también coincide con los resultados de Muñoz et al. (2012), quienes al ajustar los modelos de Schumacher y Meyer obtuvieron valores idénticos para la predicción de volúmenes de fuste total para plantaciones de Pinus greggii Engelm. $\left(\mathrm{R}^{2}\right.$ adj $\left.=0.98\right)$, con árboles de diferentes edades y un intervalo de valores del diámetro normal de $5 \mathrm{~cm}$ a $55 \mathrm{~cm}$. Sus hallazgos presentaron una mayor eficiencia pues utilizaron un mayor intervalo de categorías diamétricas y edades, lo que proporciona una mayor precisión en los resultados.

Armijos (2013) aplicó la ecuación lineal múltiple en la elaboración de tablas de volumen local de doble entrada para Tectona grandis en rodales de 13 años. Este autor, concluye que esta ecuación puede ser aplicada en otras plantaciones con condiciones similares al sitio de la plantación.

Utilizando el Modelo de Meyer (1941) es posible calcular de manera más precisa el volumen fustal en plantaciones de teca como lo demostraron Camacho y Madrigal (1997) y Torres (2004), quienes utilizaron modelos matemáticos para la estimación del volumen fustal.

Por ello es importante el ajuste y selección de modelos matemáticos que permitan predecir el volumen fustal en plantaciones forestales comerciales con condiciones diferentes de sitio y manejo silvícola empleado. 
Es viable desarrollar con mayor amplitud esta investigación mediante la aplicación de un esquema de índice de sitio para calificar la productividad de los terrenos donde se establece la plantación, tal como los estudios de Camacho et al. (2013), que generaron ecuaciones de crecimiento en diámetro, altura y volumen. O bien, un sistema de cubicación para árboles individuales mediante funciones compatibles de ahusamiento-volumen, como lo realizaron Tamarit et al. (2014).

Se recomienda incorporar el Modelo de Meyer (1941) a los sistemas de crecimiento y productividad, con la finalidad de realizar determinaciones más precisas del volumen existente en plantaciones de Tectona grandis con condiciones físicas (altitud, suelo, clima) y biológicas (edad) parecidas a las del sitio de este estudio en el estado de Michoacán.

\section{CONCLUSIONES}

Se recomienda el uso del M4 para la predicción del volumen fustal en función del diámetro normal $(1.30 \mathrm{~m})$ y la altura total de Tectona grandis en plantaciones del estado de Michoacán con condiciones edafoclimáticas similares.

El modelo ajustado representa una importante herramienta que puede ser usada para la cuantificación de volumen fustal, permite la extrapolación a la plantación de forma más precisa en comparación con las mediciones necesarias para cubicar un árbol y calcular su volumen las cuales pueden ser costosas y lentas.

\section{REFERENCIAS}

Allison, P. D. (2010). Survival analysis using SAS: A practical guide. Sas Institute.

Álvarez, J. G., Barrio, M., Diéguez, U., \& Rojo, A. (2003). Metodología para la construcción de curvas de calidad de estación. Cuadernos de La Sociedad Española de Ciencias Forestales, 309(1), 303-309.

Armijos, D. D. (2013). Construcción de tablas volumétricas y cálculo de factor de forma (FF.) para dos especies, teca (Tectona grandis) y melina (Gmelina arborea) en tres plantaciones de la empresa Reybanpac CA en la Provincia de los Ríos. Facultad de Recursos Naturales Escuela de
Ingeniería Forestal, Riobamba, Ecuador. Recuperado de http://dspace.espoch.edu.ec/handle/123456789/2967

Barrios, A., López, A. M., \& Nieto, V. (2014). Predicción de volúmenes comerciales de Eucalyptus grandis a través de modelos de volumen total y de razón. Colombia Forestal, 17(2), 137-149.

Burkhart, H. E. (1977). Cubic-foot volume of loblolly pine to any height limit. Southern Journal of Applied Forestry, 1(2), 7-9, 166-168. doi: 10.1093/sjaf/4.4.166

Camacho-Linton, A., Ramírez-Maldonado, H., de los SantosPosadas, H. M., \& Zamudio-Sánchez, F. J. (2013). Tablas de rendimiento para teca (Tectona grandis $\mathrm{L}$.) en el Estado de Campeche. Revista Mexicana de Ciencias Forestales, 4(19), 92 101.

Camacho, P., \& Madrigal, T. (1997). Ecuaciones de volumen preliminares para Tectona grandis. En 3. Congreso Forestal Nacional. 27-29 Agosto 1997. No. 634.9097286063 C749 1997. (pp. 131-133). San José, Costa Rica.

Comisión Nacional Forestal, [CONAFOR]. (2014). México cuenta con 270 mil hectáreas de Plantaciones Forestales Comerciales. México D. F.: Semarnat.

Corona, I. G., Jacas, D. F., Tamayo, M. F., \& Pereira, E. A. (1999). Índice de sitio y tablas de crecimiento para plantaciones jóvenes de Tectona grandis L . en la Unidad silvícola Guisa . Granma, $15 . \quad$ Recuperado de https://www.buscagro.com/biblioteca/PinardelRio/Tect onaGrandis.pdf

Da Cunha, T. A., Vargas, M. J. O., \& Escalier, H. M. (2009). Ajuste y selección de modelos de regresión para estimar el volumen total de árboles. Documento Técnico, (5), 27. Recuperado de https://www.researchgate.net/profile/Thiago_Da_Cunh a2/publication/236657148_Ajuste_y_seleccion_de_mode los_de_regresion_para_estimar_el_volumen_total_de_arb oles_Autores_Thiago_Augusto_da_Cunha_Cochabamba _-Bolivia/links/0deec518b99cc23eed000000.pdf

De Cesaro, A., Engel, O. A., Finger, C. A., \& Schneider, P. R. (1994). Comparação dos métodos de amostragem de área fixa, relascopia, e de seis árvores, quanto a eficiência, no inventário florestal de um povoamento de Pinus sp. Ciência Florestal, 4(1), 97-108. Recuperado de http://coral.ufsm.br/cienciaflorestal/artigos/v4n1/art6v $4 n 1 . p d f$

Draper, N. R., \& Smith, H. (2014). Applied regression analysis. John Wiley \& Sons. Recuperado de https:/ / books.google.com.mx / books?hl=es\&lr=\&id=uS 
ReBAAAQBAJ\&oi=fnd\&pg=PT12\&dq=applied +regress ion + analysis + draper\&ots $=\mathrm{Pa} 8 \mathrm{xAJSerZ \& sig}=\mathrm{WM} 7 \mathrm{wL} 8 \mathrm{H}$ i_MUUeQQPkVCrnHz_dYA

Emanuelli, P., \& Milla, F. (2014). Construcción de funciones de volumen. Volumen, Biomasa y Carbono Forestal, 4, 56. Recuperado de http://190.57.68.218:8080/jspui/handle/123456789/50

Husch, B., Beers, T. W., \& Kershaw Jr, J. A. (2002). Forest Mensuration and Statistics. U.S.A.: Ronald Press. Recuperado de https://books.google.com.mx/books?hl=es\&lr=\&id=p0 v3m8Pau$\mathrm{kC \& oi}=$ fnd\&pg $=$ PR11\&dq $=$ Forest + Mensuration + and + Statistics\&ots $=$ LHHJqoHxZv\&sig $=5 \mathrm{v} 0 \mathrm{kRTHiaRHWV2}$ AvP5M3o06qy24

Hynynen, J. (2011). Conceptos básicos para la modelación del crecimiento forestal. Recursos Naturales Y Ambiente, (64), 2227.

Instituto Nacional De Estadística Geografía e Informática, [INEGI]. (2009). Prontuario de información geográfica municipal de los Estados Unidos Mexicanos. México D. F.: Inegi.

Louppe, D., \& Mille, G. (2015). Mémento du forestier tropical. France. Editions Quæ. Recuperado de http://www.quae.com/fr/r4730-memento-du-forestiertropical.html

Meyer, H. A. (1941). A correction for a syistematic error occurring in the application of the logarithmic volume equation. Pensylvania Forest School Research. Paper, 7(3).

Muñoz-Flores, H. J., Velarde-Ramírez, J. C., García-Magaña, J. J., Sáenz-Reyes, J., Olvera-Delgadillo, E. H., \& HernándezRamos, J. (2012). Predicción de volúmenes de fuste total para plantaciones de Pinus greggii Engelm. Revista Mexicana de Ciencias Forestales, 3(14), 11-24.

Naslud, M. (1936). Skogsförsöksanstanltens gallringsförsök I tallskog primärbearbetning. Bulletin De L'Institut D’Expermientation Forestiere de Suede. Stockholm. Recuperado de https://pub.epsilon.slu.se/10159/1/medd_statens_skogs forskningsanst_029_01.pdf

Parresol, B. R. (1999). Assessing tree and stand biomass: a review with examples and critical comparisons. Forest Science, 45(4), 573-593.
Pérez, L. D., \& Kanninen, M. (2007). Estimacion del volumen comercial a diámetros y alturas variables para Tectona grandis L F en Costa Rica. Recursos Naturales Y Ambiente, (49-50).

Prodan, M., Peters, R., Cox, F., \& Real, P. (1997). Mensura forestal. Recuperado de http://agris.fao.org/agrissearch/search.do?recordID $=$ XF2016033393

Reyes, J. R. (2006). Sistema de cubicación para Eucalyptus grandis y E. urophylla en los límites de Veracruz y Oaxaca. Universidad Autónoma Chapingo División de Ciencias Forestales, Texcoco, Estado de México.

Sakici, O. E., Misir, N., Yavuz, H., \& Misir, M. (2008). Stem taper functions for Abies nordmanniana subsp. bornmulleriana in Turkey. Scandinavian Journal of Forest Research, 23(6), 522533. doi: 10.1080/02827580802552453

Schlaegel, B. E. (1982). Notes: Boxelder (Acer negundo L.) Biomass Component Regression Analysis for the Mississippi Delta. Forest Science, (28), 355-358. doi: 10.1093 / forestscience/28.2.355

Segura, M., \& Andrade, H. J. (2008). ¿Cómo construir modelos alométricos de volumen, biomasa o carbono de especies leñosas perennes? Agroforestería En Las Américas, 46, 89-96. Recuperado de http://201.207.189.89/handle/11554/6935

Stoate, T. N. (1945). The use of a volumen equation in pine stands. Australian Forestry, 9(1), 48-52. doi: 10.1080/00049158.1945.10675220

Tamarit-Urías, J. C., De los Santos-Posadas, H. M., Aldrete, M., Valdez-Lazarde, J. R., Ramírez-Maldonado, H., \& Guerra de la Cruz, V. (2014). Sistema de cubicación para árboles individuales de Tectona grandis L. f. mediante funciones compatibles de ahusamiento-volumen. Revista Mexicana de Ciencias Forestales, 5(21), 58-74. Recuperado de http://www.scielo.org.mx/scielo.php?pid=S2007$11322014000100005 \&$ script $=$ sci_arttext\&tlng=en

Tedeschi, L. O. (2006). Assessment of the adequacy of mathematical models. Agricultural Systems, 89(2-3), 225247. doi: 10.1016/j.agsy.2005.11.004

Torres, D. A. (2004). Modelación del Crecimiento y Producción en Volumen y Biomasa de la Teca. Universidad Nacional de Colombia Sede Medellín, Medellín.

Vallejo, Á., \& Avendaño, J. (2013). Modelos de crecimiento y rendimiento. En R. de Camino \& J. Pierre (Eds.), Las plantaciones de teca en América Latina: Mitos y realidades (pp. 114-133). Turrualba, C.R. 
Manuscrito recibido el 18 de abril de 2017

Aceptado el 21 de mayo de 2018

Publicado el 4 de diciembre de 2018

Este documento se debe citar como:

Telles A., R, Gómez C., M., Alanís-Rodríguez, E., AguirreCalderón, O. A., \& Jiménez Pérez, J. (2018). Ajuste y selección de modelos matemáticos para predecir el volumen fustal de Tectona grandis L. f. en Nuevo Urecho, Michoacán, México. Madera y Bosques, 24(3), e2431544. doi: 10.21829/myb.2018.2431544

Madera y Bosques por Instituto de Ecología, A.C. se distribuye bajo una Licencia Creative Commons Atribución-NoComercialCompartirlgual 4.0 Internacional. 